\title{
Solution of $n$.th Order Constant Coefficients Complex Partial Derivative Equations by Using Fourier Transform Method
}

\author{
Murat Düz*
}

\begin{abstract}
The aim of this article is to find a specific solution for constant coefficients complex partial differential equations using Fourier transform. Firstly, equality of complex derivatives have been obtained from kind real derivatives. Later Fourier Transforms have been used for obtained equation. Finally a formula has been given for a special solution of these kind equations. Also, examples are given to display the validity of the present method.
\end{abstract}

Keywords: Complex equation; Fourier Transform; Special solution.

AMS Subject Classification (2020): Primary: 34B10 ; Secondary: $34 B 15$.

${ }^{*}$ Corresponding author

\section{Introduction}

There is no real solution of some algebraic equations. For example $x^{2}+1=0$ equation has no to a solution in $\mathrm{R}$. Similarly general solutions of some differential equations in real space can not be found, too. For example

$$
U_{x x}+U_{y y}=0 .
$$

Laplace equation does not have general solution in $R^{2}$. But

$$
U_{z \bar{z}}=0 .
$$

which is equivalent to (1.1) Laplace equation has general solution in complex space and the solution of (1.2) is $U=f(z)+g(\bar{z})$, where $g$ is anti-analytic, $f$ is analytic and $z=x+i y$. That is, although an equation doesn't have a general solution in real, it can have general solution in complex space. The existence of solution of complex differential equations, exact and numerical solution methods are important research topics for mathematicians. The most basic works in the theory of complex differential equations are "Theory of Pseudo Analytic Functions" which is written by [2] and "Generalized Analytic Functions" which is written by [11]. There are also recently studies on complex differential equations. Complex differential equations in the form $f^{\prime \prime}(z)+A(z) f(z)=0$ have been studied in $[5,6,9]$. Approximate solutions of linear complex differential equations which have variable coefficients were studied in [9] and [10]. Similarly numerical solutions of linear complex differential equations have been studied with complex operational matrix method in [12]. In studies [13] and [14], the numerical solutions of linear complex differential equations have been obtained by using Legendre and Hermite polynomials. In this study, the equations

Received : 21-11-2019, Accepted : 20-09-2020 
of the following form has been studied.

$$
\begin{aligned}
& A_{n, 0} \frac{\partial^{n} w}{\partial z^{n}}+A_{n-1,1} \frac{\partial^{n} w}{\partial z^{n-1} \partial \bar{z}}+\ldots+A_{0, n} \frac{\partial^{n} w}{\partial \bar{z}^{n}}+A_{n-1,0} \frac{\partial^{n-1} w}{\partial z^{n-1}}+A_{n-2,1} \frac{\partial^{n-1} w}{\partial z^{n-2} \partial \bar{z}}+\ldots+A_{0, n-1} \frac{\partial^{n-1} w}{\partial \bar{z}^{n-1}}+\ldots \\
& +A_{1,0} \frac{\partial w}{\partial z}+A_{0,1} \frac{\partial w}{\partial \bar{z}}+A_{0,0} w=F(z, \bar{z}) .
\end{aligned}
$$

Where $A_{i, j}(1 \leq i \leq n, 1 \leq j \leq n)$ are real constants. Fourier transform has been used for solution . Fourier transform is an important integral transform. Integral transform is a method such that it is used for solutions of linear differential equations. Integral transforms are transformations that transform one function into another function under integral. These transformations are in the following form

$$
g(\alpha)=\int_{a}^{b} f(t) \cdot K(\alpha, t) d t
$$

where $K(\alpha, t)$ is called the kernel of the transform. In (1.4), $f(t)$ is input function and $g(\alpha)$ is output function. Integral transforms have been used to solve many problems in mathematical physics, applied mathematics and engineering sciences. The Fourier Transform was given by Jean-Baptiste Joseph Fourier. This transform is used in many areas of engineering and it has been very useful for analyzing harmonic signals. The sound analysis can be given as example to applications of Fourier transform. The problem of finding who a sound belongs to can be given as an example of the applications of Fourier transform. The Fourier transform is also very useful in mathematics. Differential equations, generalized integrals, integral equations can be computed with the help of Fourier transforms. For example, it can be seen with aid Fourier transform of

$$
f(t)=\left\{\begin{array}{cc}
1 & ;-1<t<1 \\
0 & ; t \in \mathbb{R}-[-1,1]
\end{array}\right.
$$

function that $\int_{0}^{\infty} \frac{\sin t}{t} d t=\frac{\pi}{2}$.

Linear complex partial derivative equations with constant coefficients from first order has been solved by using Fourier transform in [8]. This study is a generalization of [8]. A theorem has been given for (1.3) differential equation. This work has been organized as follows: Basic definitions and theorems are given in Section 2. In the next section, we mention that the relationship between complex derivatives and real derivatives. Then, a formulation to solution of the equation (1.3) has been obtained and some examples are given for validity of the method. In final section, conclusion is given.

\section{Basic Definitions and Theorems}

Definition 2.1. Fourier transform of $f(t)$ is defined as

$$
\mathcal{F}[f(t)]=\int_{-\infty}^{\infty} f(t) e^{-i w t} d t
$$

As integral (2.1) is function of $w$

$$
\mathcal{F}[f(t)]=F(w)
$$

is written.

Definition 2.2. If $\mathcal{F}[f(t)]=F(w)$ then $f(t)$ is called inverse Fourier transform of $F(w)$, where

$$
f(t)=\frac{1}{2 \pi} \int_{-\infty}^{\infty} F(w) e^{i w t} d w
$$

and it is denoted by $f(t)=\mathcal{F}^{-1}[F(w)]$.

Theorem 2.1. [1] and [3] The Fourier Transform is linear. Let $c_{1}, c_{2} \in \mathbb{R}$. Then,

$$
\mathcal{F}\left[c_{1} f_{1}(t)+c_{2} f_{2}(t)\right]=c_{1} \mathcal{F}\left[f_{1}(t)\right]+c_{2} \mathcal{F}\left[f_{2}(t)\right] .
$$


Theorem 2.2. [1] and [3] Let $f(t)$ be continuous or partly continuous in the interval $(-\infty, \infty)$ and $f(t), f^{\prime}(t), \ldots, f^{(n-1)}(t) \rightarrow$ 0 for $|t| \rightarrow \infty$. If $f, f^{\prime}, \ldots, f^{(n-1)}$ are absolutely integrable in the interval $(-\infty, \infty)$, then

$$
\mathcal{F}\left[f^{(n)}(t)\right]=(i w)^{n} F(w)
$$

Now let's give the definition of the Dirac delta function, which is a commonly used function in Fourier transform.

$$
\delta(t)=\left\{\begin{array}{cc}
0 & ; t \neq 0 \\
\infty & ; t=0
\end{array}\right.
$$

The Dirac delta function has some following properties [3] and [4].

$$
\begin{aligned}
& \int_{-\infty}^{\infty} \delta(t) d t=1, \\
& \int_{-\infty}^{\infty} f(t) \delta\left(t-t_{0}\right) d t=f\left(t_{0}\right), \\
& \left(w-w_{0}\right)^{n} \delta^{(n)}\left(w-w_{0}\right)=(-1)^{n} n ! \delta\left(w-w_{0}\right), \\
& \int_{-\infty}^{\infty} \frac{\delta\left(w-w_{0}\right)}{\left(w-w_{0}\right)^{n}} f(w) d w=\frac{1}{n !} \frac{d^{n} f(w)}{d w^{n}}\left(w=w_{0}\right) .
\end{aligned}
$$

Theorem 2.3. [1] and [3]. Fourier transforms of some functions are following:

(i) $\mathcal{F}[1]=2 \pi \delta(w)$,

(ii) $\mathcal{F}\left[t^{n}\right]=2 \pi i^{n} \delta^{(n)}(w)$,

(iii) $\mathcal{F}\left[t^{n} \cdot y\right]=i^{n} \frac{d^{n} \mathcal{F}[y]}{d w^{n}}$,

(iv) $\mathcal{F}\left[e^{i w_{0} t}\right]=2 \pi \delta\left(w-w_{0}\right)$,

(v) If $\mathcal{F}[y]=Y(w)$, then $\mathcal{F}\left[e^{i w_{0} t} \cdot y\right]=Y\left(w-w_{0}\right)$,

(vi) $\mathcal{F}\left[e^{a t}\right]=2 \pi \delta(w+i a)$,

(vii) If $\mathcal{F}[y]=Y(w)$ then $\mathcal{F}\left[e^{w_{0} t} \cdot y\right]=Y\left(w+i w_{0}\right)$.

(viii) $\mathcal{F}[\delta(t)]=1$.

Lemma 2.1. [7]

Let $f(x, y)$ be continuous or partly continuous in the interval $(-\infty, \infty)$ and $f(x, y), \frac{\partial f(x, y)}{\partial x}, \frac{\partial^{2} f(x, y)}{\partial x^{2}}, \ldots, \frac{\partial^{n-1} f(x, y)}{\partial x^{n-1}} \rightarrow$ 0 for $|x| \rightarrow \infty$. If $f(x, y), \frac{\partial f(x, y)}{\partial x}, \frac{\partial^{2} f(x, y)}{\partial x^{2}}, \ldots, \frac{\partial^{n-1} f(x, y)}{\partial x^{n-1}}$ are absolutely integrable in $(-\infty, \infty)$, then Fourier transforms of partial derivatives $n$.th order of $f(x, y)$ are following:

(i) $\mathcal{F}\left(\frac{\partial^{n} f(x, y)}{\partial x^{n}}\right)=(i w)^{n} F(w, y)$,

(ii) $\mathcal{F}\left(\frac{\partial^{n} f(x, y)}{\partial y^{n}}\right)=\frac{\partial^{n} F(w, y)}{\partial y^{n}}$,

where is $F(w, y)=\mathcal{F}(f(x, y))$.

Lemma 2.2. [7] Fourier transforms of partial derivatives $(n+m)$.th order of $f(x, y)$ are following:

$$
\mathcal{F}\left[\frac{\partial^{n+m} f(x, y)}{\partial x^{n} \partial y^{m}}\right]=(i w)^{n} \frac{\partial^{m} F(w, y)}{\partial y^{m}} .
$$




\section{Equivalent of Complex Derivatives in the Real Derivatives}

Definition 3.1. Derivative operators

$$
\frac{\partial}{\partial z}=\frac{1}{2}\left(\frac{\partial}{\partial x}-i \frac{\partial}{\partial y}\right), \frac{\partial}{\partial \bar{z}}=\frac{1}{2}\left(\frac{\partial}{\partial x}+i \frac{\partial}{\partial y}\right) .
$$

are called complex derivative operators.

Lemma 3.1. Let $n$ and $r$ be a positive integer number and $n \geq r$, then

$$
\left(\begin{array}{l}
n \\
r
\end{array}\right)+\left(\begin{array}{c}
n \\
r-1
\end{array}\right)=\left(\begin{array}{c}
n+1 \\
r
\end{array}\right)
$$

Lemma 3.2. From sum symbols following equality is valid:

$$
\sum_{k=0}^{n} a_{k} \sum_{h=0}^{r} b_{h}=\sum_{k=0}^{n} \sum_{h=0}^{r} a_{k} b_{h}
$$

Lemma 3.3. Let $w=w(z, \bar{z})$ be a complex variables and valued function. Then,

$$
\frac{\partial^{n} w}{\partial z^{n}}=\frac{1}{2^{n}} \sum_{k=0}^{n}(-i)^{k}\left(\begin{array}{l}
n \\
k
\end{array}\right) \frac{\partial^{n} w}{\partial x^{n-k} \partial y^{k}} .
$$

Proof. Proof can be made by induction. For $n=1$, due to equality then we have

$$
\frac{\partial w}{\partial z}=\frac{1}{2}\left(\frac{\partial w}{\partial x}-i \frac{\partial w}{\partial y}\right)=\frac{1}{2^{1}}\left[(-i)^{0}\left(\begin{array}{l}
1 \\
0
\end{array}\right) \frac{\partial w}{\partial x}++(-i)^{1}\left(\begin{array}{l}
1 \\
1
\end{array}\right) \frac{\partial w}{\partial y}\right]=\frac{1}{2} \sum_{k=0}^{1}(-i)^{k}\left(\begin{array}{l}
1 \\
k
\end{array}\right) \frac{\partial w}{\partial x^{1-k} \partial y^{k}} .
$$

As a result, it is true for $n=1$. Let $n=r$ is true. So, we have

$$
\frac{\partial^{r} w}{\partial z^{r}}=\frac{1}{2^{r}} \sum_{k=0}^{r}(-i)^{k}\left(\begin{array}{l}
r \\
k
\end{array}\right) \frac{\partial^{r} w}{\partial x^{r-k} \partial y^{k}} .
$$

Then, we must show that it is true for $n=r+1$.

$$
\begin{aligned}
& \frac{\partial^{r+1} w}{\partial z^{r+1}}=\frac{\partial}{\partial z} \frac{\partial^{r} w}{\partial z^{r}}=\frac{\partial}{\partial z} \frac{1}{2^{r}} \sum_{k=0}^{r}(-i)^{k}\left(\begin{array}{l}
r \\
k
\end{array}\right) \frac{\partial^{r} w}{\partial x^{r-k} \partial y^{k}}=\frac{1}{2} \frac{\partial}{\partial x}\left(\frac{1}{2^{r}} \sum_{k=0}^{r}(-i)^{k}\left(\begin{array}{l}
r \\
k
\end{array}\right) \frac{\partial^{r} w}{\partial x^{r-k} \partial y^{k}}\right)- \\
& i \frac{1}{2} \frac{\partial}{\partial y}\left(\frac{1}{2^{r}} \sum_{k=0}^{r}(-i)^{k}\left(\begin{array}{l}
r \\
k
\end{array}\right) \frac{\partial^{r} w}{\partial x^{r-k} \partial y^{k}}\right)=\frac{1}{2^{r+1}} \sum_{k=0}^{r}(-i)^{k}\left(\begin{array}{l}
r \\
k
\end{array}\right) \frac{\partial^{r+1} w}{\partial x^{r+1-k} \partial y^{k}} \\
& -i \frac{1}{2^{r+1}} \sum_{k=0}^{r}(-i)^{k}\left(\begin{array}{l}
r \\
k
\end{array}\right) \frac{\partial^{r+1} w}{\partial x^{r-k} \partial y^{k+1}}=\frac{1}{2^{r+1}}\left[\left(\frac{\partial^{r+1} w}{\partial x^{r+1}}-i \frac{\partial^{r+1} w}{\partial x^{r} \partial y}\right)+\left(-i\left(\begin{array}{l}
r \\
1
\end{array}\right) \frac{\partial^{r+1} w}{\partial x^{r} \partial y}+(-i)^{2}\left(\begin{array}{c}
r \\
1
\end{array}\right) \frac{\partial^{r+1} w}{\partial x^{r-1} \partial y^{2}}\right)\right] \\
& +\frac{1}{2^{r+1}}\left[+\ldots+(-i)^{r}\left(\begin{array}{l}
r \\
r
\end{array}\right)\left(\frac{\partial^{r+1} w}{\partial x \partial y^{r}}-i \frac{\partial^{r+1} w}{r i g h t \partial y^{r+1}}\right)\right] .
\end{aligned}
$$

If we take the common multiplier in paranthesis, than we get following equality

$$
\begin{aligned}
& =\frac{1}{2^{r+1}}\left[\frac{\partial^{r+1} w}{\partial x^{r+1}}-i\left(\frac{\partial^{r+1} w}{\partial x^{r} \partial y}+\left(\begin{array}{l}
r \\
1
\end{array}\right) \frac{\partial^{r+1} w}{\partial x^{r} \partial y}\right)+(-i)^{2}\left(\left(\begin{array}{c}
r \\
1
\end{array}\right) \frac{\partial^{r+1} w}{\partial x^{r-1} \partial y^{2}}+\left(\begin{array}{c}
r \\
2
\end{array}\right) \frac{\partial^{r+1} w}{\partial x^{r-1} \partial y^{2}}\right)\right] \\
& =\frac{1}{2^{r+1}}\left[\frac{\partial^{r+1} w}{\partial x^{r+1}}-i\left(\frac{\partial^{r+1} w}{\partial x^{r} \partial y}+\left(\begin{array}{l}
r \\
1
\end{array}\right) \frac{\partial^{r+1} w}{\partial x^{r} \partial y}\right)+(-i)^{2}\left(\left(\begin{array}{c}
r \\
1
\end{array}\right) \frac{\partial^{r+1} w}{\partial x^{r-1} \partial y^{2}}+\left(\begin{array}{l}
r \\
2
\end{array}\right) \frac{\partial^{r+1} w}{\partial x^{r-1} \partial y^{2}}\right)\right] \\
& +\frac{1}{2^{r+1}}\left[+\ldots+(-i)^{r}\left(\left(\begin{array}{c}
r \\
r-1
\end{array}\right) \frac{\partial^{r+1} w}{\partial x \partial y^{r}}+\left(\begin{array}{l}
r \\
r
\end{array}\right) \frac{\partial^{r+1} w}{\partial x \partial y^{r}}\right)+(-i)^{r+1} \frac{\partial^{r+1} w}{\partial y^{r+1}}\right] .
\end{aligned}
$$




$$
\begin{aligned}
& \text { From Lemma } 3.1 \\
& =\frac{1}{2^{r+1}}\left[\frac{\partial^{r+1} w}{\partial x^{r+1}}-i\left(\begin{array}{c}
r+1 \\
1
\end{array}\right) \frac{\partial^{r+1} w}{\partial x^{r} \partial y}+(-i)^{2}\left(\begin{array}{c}
r+1 \\
2
\end{array}\right) \frac{\partial^{r+1} w}{\partial x^{r-1} \partial y^{2}}\right] \\
& +\frac{1}{2^{r+1}}\left[+\ldots+(-i)^{r}\left(\begin{array}{c}
r+1 \\
r
\end{array}\right) \frac{\partial^{r+1} w}{\partial x \partial y^{r}}+(-i)^{r+1}\left(\begin{array}{c}
r+1 \\
r+1
\end{array}\right) \frac{\partial^{r+1} w}{\partial y^{r+1}}\right] \\
& =\frac{1}{2^{r+1}} \sum_{k=0}^{r+1}(-i)^{k}\left(\begin{array}{c}
r+1 \\
k
\end{array}\right) \frac{\partial^{r+1} w}{\partial x^{r+1-k} \partial y^{k}} .
\end{aligned}
$$

As a result proof is completed.

Lemma 3.4. Let $w=w(z, \bar{z})$ be a complex variables and valued function. Then,

$$
\frac{\partial^{n} w}{\partial \bar{z}^{n}}=\frac{1}{2^{n}} \sum_{k=0}^{n}(i)^{k}\left(\begin{array}{l}
n \\
k
\end{array}\right) \frac{\partial^{n} w}{\partial x^{n-k} \partial y^{k}} .
$$

Proof. The proof is similar to proof of the Lemma 3.3.

Theorem 3.1. Let $w=w(z, \bar{z})$ be a complex variables and valued function. Then,

$$
\frac{\partial^{n+r} w}{\partial z^{n} \partial \bar{z}^{r}}=\frac{1}{2^{n+r}} \sum_{h=0}^{n} \sum_{k=0}^{r}(-i)^{h} i^{k}\left(\begin{array}{l}
n \\
h
\end{array}\right)\left(\begin{array}{l}
r \\
k
\end{array}\right) \frac{\partial^{n+r} w}{\partial x^{n+r-(h+k)} \partial y^{h+k}} .
$$

Proof.

$$
\begin{aligned}
& \frac{\partial^{n+r} w}{\partial z^{n} \partial \bar{z}^{r}}=\frac{\partial^{n}}{\partial z^{n}} \frac{\partial^{r} w}{\partial \bar{z}^{r}}=\frac{\partial^{n}}{\partial z^{n}}\left(\frac{1}{2^{r}} \sum_{k=0}^{r}(i)^{k}\left(\begin{array}{l}
r \\
k
\end{array}\right) \frac{\partial^{r} w}{\partial x^{r-k} \partial y^{k}}\right)=\frac{1}{2^{n+r}} \sum_{h=0}^{n}(-i)^{h}\left(\begin{array}{l}
n \\
h
\end{array}\right) \frac{\partial^{n}}{\partial x^{n-h} \partial y^{h}}\left(\sum_{k=0}^{r}(i)^{k}\left(\begin{array}{l}
r \\
k
\end{array}\right) \frac{\partial^{r} w}{\partial x^{r-k} \partial y^{k}}\right) \\
& =\frac{1}{2^{n+r}} \sum_{h=0}^{n} \sum_{k=0}^{r}(-i)^{h}(i)^{k}\left(\begin{array}{l}
n \\
h
\end{array}\right)\left(\begin{array}{l}
r \\
k
\end{array}\right) \frac{\partial^{n+r} w}{\partial x^{n+r-(h+k)} \partial y^{h+k}} .
\end{aligned}
$$

Theorem 3.2. Let $A_{i, j}$ are real constants $(1 \leq i \leq n, 1 \leq j \leq n, i+j \leq n), w=w(z, \bar{z})$ is a polynomial of $z, \bar{z}$. Then a special solution of

$$
\begin{aligned}
& A_{n, 0} \frac{\partial^{n} w}{\partial z^{n}}+A_{n-1,1} \frac{\partial^{n} w}{\partial z^{n-1} \partial \bar{z}}+\ldots+A_{0, n} \frac{\partial^{n} w}{\partial \bar{z}^{n}}+A_{n-1,0} \frac{\partial^{n-1} w}{\partial z^{n-1}}+A_{n-2,1} \frac{\partial^{n-1} w}{\partial z^{n-2} \partial \bar{z}}+\ldots+ \\
& A_{0, n-1} \frac{\partial^{n-1} w}{\partial \bar{z}^{n-1}}+\ldots+A_{1,0} \frac{\partial w}{\partial z}+A_{0,1} \frac{\partial w}{\partial \bar{z}}+A_{0,0} w=F(z, \bar{z})
\end{aligned}
$$

is

$$
w(z, \bar{z})=\mathcal{F}^{-1}\left[\frac{\mathcal{F}(F(z, \bar{z}))}{\sum_{k=0}^{n} \sum_{l=0}^{n-k} A_{n-k-l, l} \frac{1}{2^{n-k}} \sum_{m=0}^{n-k-l} \sum_{h=0}^{l} P(w, D)}\right] \text {, }
$$

where $P(w, D)=(-i)^{m} i^{h}\left(\begin{array}{c}n-l-k \\ m\end{array}\right)\left(\begin{array}{l}l \\ h\end{array}\right)(i w)^{n-m-h} D^{h+m}$.

Proof. Using Theorem 3.1, the above equation which has been given in theorem can be written from kind of real derivatives as follows:

$$
\begin{aligned}
& A_{n, 0} \frac{1}{2^{n}} \sum_{k=0}^{n}(-i)^{k}\left(\begin{array}{l}
n \\
k
\end{array}\right) \frac{\partial^{n} w}{\partial x^{n-k} \partial y^{k}}+A_{n-1,1} \frac{1}{2^{n}} \sum_{h=0}^{n-1} \sum_{k=0}^{1}(-i)^{h} i^{k}\left(\begin{array}{c}
n-1 \\
h
\end{array}\right) \frac{\partial^{n} w}{\partial x^{n-h-k} \partial y^{h+k}}+\ldots+ \\
& A_{0, n} \frac{1}{2^{n}} \sum_{k=0}^{n} i^{k}\left(\begin{array}{l}
n \\
k
\end{array}\right) \frac{\partial^{n} w}{\partial x^{n-k} \partial y^{k}}+A_{n-1,0} \frac{1}{2^{n-1}} \sum_{k=0}^{n-1}(-i)^{k}\left(\begin{array}{c}
n-1 \\
k
\end{array}\right) \frac{\partial^{n-1} w}{\partial x^{n-1-k} \partial y^{k}} \\
& +A_{n-2,1} \frac{1}{2^{n-1}} \sum_{h=0}^{n-2} \sum_{k=0}^{1}(-i)^{h} i^{k}\left(\begin{array}{c}
n-2 \\
h
\end{array}\right) \frac{\partial^{n-1} w}{\partial x^{n-1-h-k} \partial y^{h+k}}+\ldots+A_{0, n-1} \frac{1}{2^{n-1}} \sum_{k=0}^{n-1} i^{k}\left(\begin{array}{c}
n-1 \\
k
\end{array}\right) \frac{\partial^{n-1} w}{\partial x^{n-1-k} \partial y^{k}} \\
& +\ldots+A_{1,0} \frac{1}{2}\left(w_{x}-i w_{y}\right)+A_{0,1} \frac{1}{2}\left(w_{x}+i w_{y}\right)+A_{0,0} w=F(z, \bar{z}) .
\end{aligned}
$$


We let apply Fourier transform for above equation.

$$
\begin{aligned}
& A_{n, 0} \frac{1}{2^{n}} \mathcal{F}\left(\sum_{k=0}^{n}(-i)^{k}\left(\begin{array}{l}
n \\
k
\end{array}\right) \frac{\partial^{n} w}{\partial x^{n-k} \partial y^{k}}\right)+\frac{A_{n-1,1}}{2^{n}} \mathcal{F}\left(\sum_{h=0}^{n-1} \sum_{k=0}^{1}(-i)^{h} i^{k}\left(\begin{array}{c}
n-1 \\
h
\end{array}\right) \frac{\partial^{n} w}{\partial x^{n-h-k} \partial y^{h+k}}\right) \\
& +\ldots+A_{0, n} \frac{1}{2^{n}} \mathcal{F}\left(\sum_{k=0}^{n} i^{k}\left(\begin{array}{l}
n \\
k
\end{array}\right) \frac{\partial^{n} w}{\partial x^{n-k} \partial y^{k}}\right)+A_{n-1,0} \frac{1}{2^{n-1}} \mathcal{F}\left(\sum_{k=0}^{n-1}(-i)^{k}\left(\begin{array}{c}
n-1 \\
k
\end{array}\right) \frac{\partial^{n-1} w}{\partial x^{n-1-k} \partial y^{k}}\right) \\
& +\frac{A_{n-2,1}}{2^{n-1}} \mathcal{F}\left(\sum_{h=0}^{n-2} \sum_{k=0}^{1}(-i)^{h} i^{k}\left(\begin{array}{c}
n-2 \\
h
\end{array}\right) \frac{\partial^{n-1} w}{\partial x^{n-1-h-k} \partial y^{h+k}}\right)+\ldots+A_{0, n-1} \frac{1}{2^{n-1}} \mathcal{F}\left(\begin{array}{c}
n-1 \\
k=0
\end{array} i^{k}\left(\begin{array}{c}
n-1 \\
k
\end{array}\right) \frac{\partial^{n-1} w}{\partial x^{n-1-k} \partial y^{k}}\right) \\
& +\ldots+A_{1,0} \frac{1}{2} \mathcal{F}\left(w_{x}-i w_{y}\right)+A_{0,1} \frac{1}{2} \mathcal{F}\left(w_{x}+i w_{y}\right)+A_{0,0} \mathcal{F}(w)=\mathcal{F}(F(z, \bar{z})) .
\end{aligned}
$$

We can write from Fourier transform of partial derivatives that:

$$
\begin{aligned}
& A_{n, 0} \frac{1}{2^{n}} \sum_{k=0}^{n}(-i)^{k}\left(\begin{array}{c}
n \\
k
\end{array}\right)(i w)^{n-k} \frac{\partial^{k} F(w, y)}{\partial y^{k}}+A_{n-1,1} \frac{1}{2^{n}} \sum_{h=0}^{n-1} \sum_{k=0}^{1}(-i)^{h} i^{k}\left(\begin{array}{c}
n-1 \\
h
\end{array}\right)(i w)^{n-h-k} \frac{\partial^{k+h} F(w, y)}{\partial y^{k+h}}+\ldots+ \\
& A_{0, n} \frac{1}{2^{n}} \sum_{k=0}^{n} i^{k}\left(\begin{array}{c}
n \\
k
\end{array}\right)(i w)^{n-k} \frac{\partial^{k} F(w, y)}{\partial y^{k}}+A_{n-1,0} \frac{1}{2^{n-1}} \sum_{k=0}^{n-1}(-i)^{k}\left(\begin{array}{c}
n-1 \\
k
\end{array}\right)(i w)^{n-1-k} \frac{\partial^{k} F(w, y)}{\partial y^{k}}+ \\
& A_{n-2,1} \frac{1}{2^{n-1}} \sum_{h=0}^{n-2} \sum_{k=0}^{1}(-i)^{h} i^{k}\left(\begin{array}{c}
n-2 \\
h
\end{array}\right)(i w)^{n-1-h-k} \frac{\partial^{k+h} F(w, y)}{\partial y^{k+h}}+\ldots+A_{0, n-1} \frac{1}{2^{n-1}} \sum_{k=0}^{n-1} i^{k}\left(\begin{array}{c}
n-1 \\
k
\end{array}\right)(i w)^{n-1-k} \frac{\partial^{k} F(w, y)}{\partial y^{k}} \\
& +A_{1,0} \frac{1}{2} \sum_{k=0}^{1}(-i)^{k}(i w)^{1-k} \frac{\partial^{k} F(w, y)}{\partial y^{k}}+A_{0,1} \frac{1}{2} \sum_{k=0}^{1} i^{k}(i w)^{1-k} \frac{\partial^{k} F(w, y)}{\partial y^{k}}+A_{0,0} F(w, y)=\mathcal{F}(F(z, \bar{z})) .
\end{aligned}
$$

We can write under a single sum if the sum of the indices is equal. In that case

$$
\begin{aligned}
& \sum_{k=0}^{n} A_{n-k, k} \frac{1}{2^{n}} \sum_{m=0}^{n-k} \sum_{h=0}^{k}(-i)^{m} i^{h}\left(\begin{array}{c}
n-k \\
m
\end{array}\right)\left(\begin{array}{l}
k \\
h
\end{array}\right)(i w)^{n-m-h} \frac{\partial^{h+m} F(w, y)}{\partial y^{h+m}}+\sum_{k=0}^{n-1} A_{n-1-k, k} \frac{1}{2^{n-1}} \sum_{m=0}^{n-1-k} \sum_{h=0}^{k}(-i)^{m} i^{h} \\
& \left(\begin{array}{c}
n-1-k \\
m
\end{array}\right)\left(\begin{array}{c}
k \\
h
\end{array}\right)(i w)^{n-1-m-h} \frac{\partial^{h+m} F(w, y)}{\partial y^{h+m}}+\ldots+\sum_{k=0}^{1} A_{1-k, k} \frac{1}{2} \sum_{m=0}^{1-k} \sum_{h=0}^{k}(-i)^{m} i^{h}\left(\begin{array}{c}
1-k \\
m
\end{array}\right)\left(\begin{array}{c}
k \\
h
\end{array}\right)(i w)^{1-m-h} \frac{\partial^{h+m} F(w, y)}{\partial y^{h+m}} \\
& +A_{0,0} F(w, y)=\mathcal{F}(F(z, \bar{z}))
\end{aligned}
$$

If we rearrange these sum symbols then we get that:

$$
\begin{gathered}
{\left[\sum_{k=0}^{n} \sum_{l=0}^{n-k} A_{n-k-l, l} \frac{1}{2^{n-k}} \sum_{m=0}^{n-k-l} \sum_{h=0}^{l}(-i)^{m} i^{h}\left(\begin{array}{c}
n-l-k \\
m
\end{array}\right)\left(\begin{array}{l}
l \\
h
\end{array}\right)(i w)^{n-m-h} D^{h+m}\right] F(w, y)=\mathcal{F}(F(z, \bar{z})),} \\
F(w, y)=\frac{\mathcal{F}(F(z, \bar{z}))}{\sum_{k=0}^{n} \sum_{l=0}^{n-k} A_{n-k-l, l} \frac{1}{2^{n-k}} \sum_{m=0}^{n-k-l} \sum_{h=0}^{l} P(w, D)}
\end{gathered}
$$

where $P(w, D)=(-i)^{m} i^{h}\left(\begin{array}{c}n-l-k \\ m\end{array}\right)\left(\begin{array}{l}l \\ h\end{array}\right)(i w)^{n-m-h} D^{h+m}$.

From inverse Fourier Transform

$$
w(z, \bar{z})=\mathcal{F}^{-1}(F(w, y))
$$

Example 3.1. Find the a special solution of following equation: 


$$
\frac{\partial^{2} w}{\partial z \partial \bar{z}}=4
$$

Solution. For the equation $n=2, A_{2,0}=A_{0,2}=A_{1,0}=A_{0,1}=A_{0,0}=0, A_{1,1}=1, F(z, \bar{z})=4$

$F(w, y)$ to be Fourier transform of $w(z, \bar{z})$, we can write that

$$
F(w, y)=\frac{4.2 \pi \delta(w)}{\frac{1}{4}\left(D^{2}-w^{2}\right)}=\frac{32 \pi \delta(w)}{-w^{2}\left(1-\frac{D^{2}}{w^{2}}\right)}=-\frac{32 \pi \delta(w)}{w^{2}}
$$

From inverse Fourier transform

$$
w(z, \bar{z})=\frac{1}{2 \pi} \int_{-\infty}^{\infty} F(w, y) e^{i w x} d w=\int_{-\infty}^{\infty} \frac{-16 \delta(w)}{w^{2}} e^{i w x} d w=-16 \frac{(i x)^{2}}{2}=8 x^{2}=8\left(\frac{z+\bar{z}}{2}\right)^{2}=2 z^{2}+4 z \bar{z}+2 \bar{z}^{2}
$$

Example 3.2. Find the a special solution of the following equation:

$$
\frac{\partial^{2} w}{\partial z^{2}}+\frac{\partial^{2} w}{\partial z \partial \bar{z}}+\frac{\partial^{2} w}{\partial \bar{z}^{2}}=2 z-3 \bar{z}
$$

Solution. For the equation $n=2, A_{2,0}=A_{0,2}=A_{1,1}=1, A_{1,0}=A_{0,1}=A_{0,0}=0, F(z, \bar{z})=2 z-3 \bar{z}$. From theorem $F(w, y)$ to be Fourier transform of $w(z, \bar{z})$, we can write that

$$
\begin{aligned}
& F(w, y)=\frac{-2 \pi i \delta^{\prime}(w)+10 \pi y i \delta(w)}{\frac{1}{4}\left[-w^{2}+2 w D-D^{2}-w^{2}-w D+w D+D^{2}-w^{2}-2 w D-D^{2}\right]} \\
& =\frac{-8 \pi i \delta^{\prime}(w)+40 \pi \delta(w)}{-3 w^{2}-D^{2}}=\frac{-8 \pi i \delta^{\prime}(w)+40 \pi y i \delta(w)}{-3 w^{2}\left(1+\frac{D^{2}}{3 w^{2}}\right)}=\frac{-8 \pi i \delta^{\prime}(w)+40 \pi i y \delta(w)}{-3 w^{2}} \\
& =\frac{-8 \pi i \delta(w)}{3 w^{3}}+\frac{40 \pi i y \delta(w)}{-3 w^{2}} .
\end{aligned}
$$

From inverse Fourier transform

$$
\begin{aligned}
& w(z, \bar{z})=\frac{1}{2 \pi} \int_{-\infty}^{\infty} F(w, y) e^{i w x} d w=\int_{-\infty}^{\infty}\left(\frac{-8 \pi i \delta(w)}{3 w^{3}}+\frac{40 \pi i y \delta(w)}{-3 w^{2}}\right) e^{i w x} d w \\
& =-\frac{4 i}{3} \int_{-\infty}^{\infty} \frac{\delta(w)}{w^{3}} e^{i w x} d w-\frac{20 i y}{3} \int_{-\infty}^{\infty} \frac{\delta(w)}{w^{2}} e^{i w x} d w=-\frac{2 x^{3}}{9}+\frac{10 y i x^{2}}{3} \\
& =-\frac{2}{9}\left(\frac{z+\bar{z}}{2}\right)^{3}+\frac{10}{3}\left(\frac{z+\bar{z}}{2}\right)^{2}\left(\frac{z-\bar{z}}{2}\right)
\end{aligned}
$$

\section{Conclusion}

In this study, it is shown that a special solution of complex partial differential equations with general constant coefficients can be found with the help of Fourier transform. In other words, an alternative way of finding a special solution for such equations is shown. The result which is obtained is given with a theorem and applied to the examples.

\section{References}

[1] Andrews, L.C., Shivamoggi, B.K.: Integral transforms for engineering. SPIE Press, (1988).

[2] Bers, L.: Theory of pseudo-analytic functions. Institute for Mathematics and Mechanics, New York, (1953).

[3] Boggess, A., Narcowıch, F.J.: A first course in wavelets with Fourier analysis. Prentice Hall, New Jersey, (2001). 
[4] Bracewell, R.N.: The Fourier transform and its applications. McGraw-HillBookCampany, Boston, (2000).

[5] Pommerenke, C.: On the mean growth of the solutions of complex linear differential equations. Complex Variables. 1 , 23-28 (1982). https:/ / doi.org/10.1080/17476938208814004

[6] Ishizaki, K.:Complex Oscillation Theory in Some Complex Domains.Journal of The Open University of Japan.32, 113-116 (2014).

[7] Düz, M.: A special solution of constant coefficients partial derivative equations with Fourier transform method. Advanced Mathematical Models \& Applications. 3 (1), 85-93 (2018).

[8] Düz, M.: Solution of complex differential equations by using Fourier transform. International Journal of Applied Mathematics. 31(1), 23-32 (2018).doi: http://dx.doi.org/10.12732/ijam.v31i1.2

[9] Gülsu, M., Sezer, M.: Approximate solution to linear complex differential equation by a new approximate approach. Applied Mathematics and Computation. 185 , 636-645 (2017). https://doi.org/10.1016/j.amc.2006.07.050

[10] Sezer, M., Tanay, B., Gülsu, M.: A Polynomial approach for solving high-order linear complex differential equations with variable coefficients in disc. Erciyes Üniversitesi Fen Bilimleri Enstitüsü Dergisi. 25 (1-2), 374-389 (2009).

[11] Vekua, I.N.: Verallgemeinerte analytische Funktionen. Akademie Verlag. Berlin, (1959).

[12] Mirzaee, F., Samadyar, N., Alipour, S.: Numerical solution of high order linear complex differential equations via complex operational matrix method. SeMA $76,1-13$ (2019). https:/ / doi.org/10.1007/s40324-018-0151-7

[13] Düşünceli, F., Çelik, E.: Numerical Solution for High-Order Linear Complex Differential Equations By Hermite Polynomials.Iğdır Univ. J. Inst. Sci. Tech. 7(4) , 189-201, (2017)

[14] Düşünceli, F., Çelik, E.: Numerical Solution for High-Order Linear Complex Differential Equations With Variable Coefficients. Numerical Methods for Partial Differential Equations. 34,(5) , 1645-1658 (2017). https://doi.org/10.1002/num.22222

\section{Affiliations}

MURAT DÜZ

ADDRESS: Karabuk University, Faculty of Sciences, Department of Mathematics, Karabuk-Turkey.

E-MAIL: mduz@karabuk.edu.tr

ORCID ID: 0000-0003-2387-4045 